\title{
Time scale for resolution of olfactory dysfunction in COVID-19*
}

\author{
Marlene M. Speth'1 Thirza Singer-Cornelius' ${ }^{1}$, Michael Oberle², \\ Isabelle Gengler ${ }^{3}$, Steffi J. Brockmeier ${ }^{1}$, Ahmad R. Sedaghat ${ }^{3}$ \\ Rhinology 58: 4, 404 - 405, 2020 \\ https://doi.org/10.4193/Rhin20.227 \\ ' Klinik für Hals-, Nasen-, Ohren- Krankheiten, Hals-und Gesichtschirurgie, Kantonsspital Aarau, Switzerland \\ *Received for publication: \\ 2 Institute for Laboratory Medicine, Kantonsspital Aarau, Aarau, Switzerland \\ May 9, 2020 \\ 3 Department of Otolaryngology - Head and Neck Surgery, University of Cincinnati College of Medicine, Cincinnati, OH, USA \\ Accepted: May 29, 2020
}

\section{To the Editor:}

By now, the 2019 novel coronavirus disease (COVID-19), caused by the SARS-CoV-2 virus, is widely recognized around the world as a pandemic that has infected millions and claimed the lives of hundreds of thousands ${ }^{(1)}$. Despite months of mitigation strategies, COVID-19 continues to spread and ascertainment of new knowledge about the disease process continues to be a priority of the medical community. Originally described by characteristic symptoms of fever, cough and/or shortness of breath that can rapidly progress to acute respiratory distress syndrome, it has become clear that COVID-19 has manifold clinical presentations ${ }^{(2-4)}$. Notably, olfactory dysfunction (OD) - decreased sense of smell-has been reported to occur in up to $85.6 \%$ of COVID-19 patients ${ }^{(2,5)}$.

The implications and long-term ramifications of OD in COVID-19 remain an area of investigation. When it occurs in COVID-19, OD appears to be an early symptom of the disease, is associated with younger age and female gender, and it tends to be severe in nature ${ }^{(6)}$. The severity of OD is reported in one series to be associated with greater severity of other COVID-19 symptoms, such as shortness of breath ${ }^{(6)}$. The nature of OD during COVID-19 has provided important insights for the disease-for example serving as a means of identifying patients early in the disease when they may be asymptomatic and potentially when new antiviral treatments may be most effective. However, the long-term ramifications of OD in COVID-19 will be equally important since these patients may present for treatment of OD. It is therefore necessary to have detailed knowledge about recovery from OD during COVID-19.

Herein we report novel data regarding resolution of OD from a previously described cohort of COVID-19 patients at Kantonsspital Aarau in Aarau, Switzerland (the characteristics of the patients are described in the cited reference) ${ }^{(6)}$. Out of a total of 112 patients who were at mean 36 days (SD: 9 days, range: 18 - 63 days) since the beginning of their COVID-19 symptoms, 66 had experienced OD at a mean time of 4 days into the COVID-19 disease course. None of the patients received any interventions for their OD. Of the patients who experienced OD as a part of COVID-19, 4.6\% reported mild, $13.8 \%$ reported moderate and $81.5 \%$ reported severe OD at its worst. Of the patients who had experienced OD symptoms, $78.8 \%$ (who had their first symptoms of COVID-19: 22 - 60 days ago) experienced complete resolution at a mean time of 11 days (SD: 9 days, median: 9.5 days, range: 2 - 36; Figure 1). However, $34.6 \%$ of patients whose OD resolved required at least 3 weeks for full resolution of the OD. In comparison, $19.7 \%$ (who had their first symptoms of COVID-19: 26 - 52 days ago) had experienced partial resolution of OD and 1.5\% (1 patient who had the first symptoms of COVID-19: 30 days ago) had no improvement of OD at all. Our results describing the time scale for resolution of $O D$ in $C O-$ VID-19 are consistent with recently reported data from a large cohort of Korean patients ${ }^{(7)}$. A Cox proportional hazard analysis was used to check for association with complete resolution of $\mathrm{OD}$ and independent variables including: severity of $\mathrm{OD}$ at its worst, timing of OD onset, age, gender, smoking history, history of allergic rhinitis, history of asthma, and severity of COVID-19 symptoms of taste loss, nasal obstruction, rhinorrhea, fever, cough and shortness of breath. Although no statistically significant relationships were found, point estimates suggestive of lower likelihood for resolution of OD were found for more severe OD (Hazard Ratio $[\mathrm{HR}]=0.64,95 \% \mathrm{Cl}: 0.39-1.05, \mathrm{p}=0.076$ ) and female gender $(\mathrm{HR}=0.59,95 \% \mathrm{Cl}: 0.34-1.03, \mathrm{p}=0.062)$. OD during COVID-19 completely resolves in the majority of patients within two weeks and $98.5 \%$ of patients had at least some improvement of OD. However, over one third of patients in our study whose OD resolved required at least 3 weeks for full resolution of OD. Moreover, we continued to see complete resolution in OD at time points beyond 1 month as well. The prognosis for recovery of OD in COVID-19 is excellent but while many patients may experience recovery within 2 weeks, there is also a sizeable fraction of patients who require weeks to over 1 month for recovery. Even as greater knowledge is obtained from longitudinal studies of COVID-19 patients with OD, our results suggest that patience and conservative management may be 


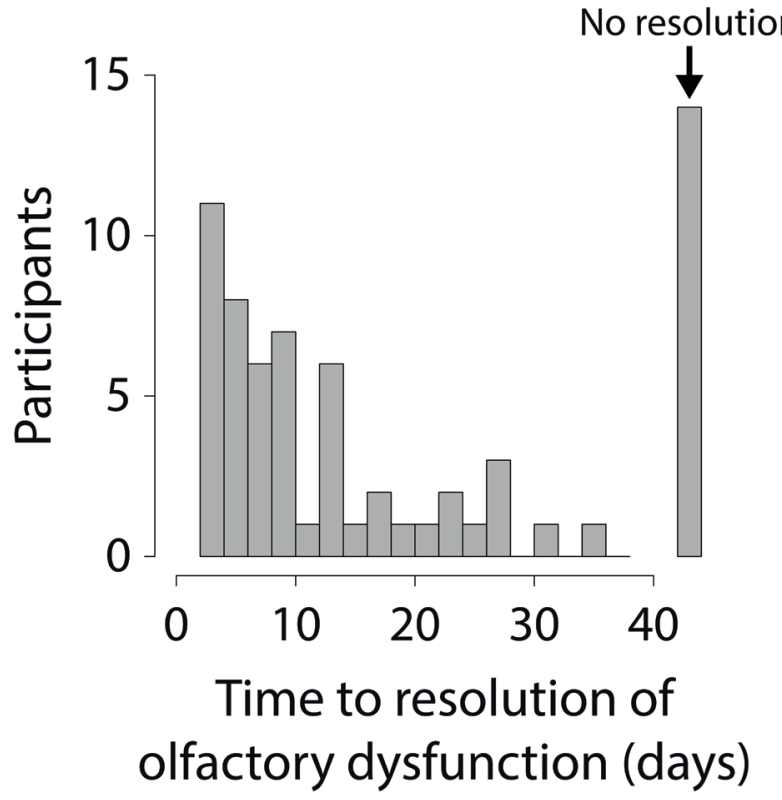

Figure 1. Histogram plot of duration of olfactory dysfunction in COVID19 patients who experienced olfactory dysfunction as a symptom. recommended for OD in COVID-19.

\section{Conflict of interest}

The authors declare no conflicts of interest.

\section{Authorship contribution}

MMS: designed and performed study, wrote and revised manuscript, approved final manuscript. TSC: designed and performed study, approved final manuscript. MO: performed study, approved final manuscript. IG: designed study, revised manuscript and approved final manuscript. SJB: designed and performed study, revised manuscript and approved final manuscript. ARS: conceived, designed and performed study, wrote and revised manuscript, approved final manuscript.

\section{Acknowledgement}

MMS and TSC received funding from Kantonsspital Aarau, Department of Otolaryngology, Funded by Research Council KSA 1410.000.128. The authors acknowledge Sofia Burgener and Vanessa Kley for their help with data collection.

\section{References}

1. Mahase E. Covid-19: WHO declares pandemic because of "alarming levels" of spread, severity, and inaction. BMJ 2020; 368:m1036.

2. Lechien JR, Chiesa-Estomba CM, De Siat DRet al. Olfactory and gustatory dysfunctions as a clinical presentation of mild-tomoderate forms of the coronavirus disease (COVID-19): a multicenter European study. Eur Arch Otorhinolaryngol 2020.

3. Lescure FX, Bouadma L, Nguyen Det al. Clinical and virological data of the first cases of COVID-19 in Europe: a case series. Lancet Infect Dis 2020.

4. Gengler I, Wang JC, Speth MM, Sedaghat AR. Sinonasal pathophysiology of SARS CoV-2 and COVID-19: a systematic review of the current evidence. Laryngoscope Investig Otolaryngol 2020. https://doi org/10.1002/lio2.384.

5. Sedaghat AR, Gengler I, Speth MM. Olfactory dysfunction: a highly prevalent symptom of COVID-19 with public health significance. Otolaryngol Head Neck Surg 2020. doi: 10.1177/0194599820926464.

6. Speth MM, Singer-Cornelius T, Oberle M, Gengler I, Brockmeier SJ, Sedaghat AR. Olfactory dysfunction and sinonasal symptomatology in COVID-19: prevalence, severity, timing and associated characteristics. Otolaryngol Head Neck Surg 2020. doi: 10.1177/0194599820929185.

7. Lee Y, Min P, Lee S, Kim SW. Prevalence and Duration of Acute Loss of Smell or Taste in COVID-19 Patients. J Korean Med Sci 2020; 35:e174.

Ahmad R. Sedaghat

Department of Otolaryngology

Head and Neck Surgery

University of Cincinnati College of

Medicine

Cincinnati, $\mathrm{OH}$

USA

E-mail: ahmad.sedaghat@uc.edu 